\title{
Antibiotic prescribing practices for hospitalised children with suspected bacterial infections in a paediatric hospital in Nigeria
}

\author{
Nneka Egbuchulam, Emmanuel N. Anyika, Rebecca O. Soremekun \\ Department of Clinical Pharmacy and Biopharmacy, Faculty of Pharmacy, University of Lagos, Nigeria
}

Received: March 26, 2018

DOI: $10.5430 /$ jha.v7n4p36

\author{
Accepted: May 16, 2018 \\ Online Published: June 6, 2018 \\ URL: https://doi.org/10.5430/jha.v7n4p36
}

\begin{abstract}
Background: The burden of infectious diseases among Nigerian children is high. These children are often prescribed antibiotics during periods of hospitalisation. Unfortunately antibiotic resistance (ABR) threatens the availability and efficacy of antibiotics for use by vulnerable children and the future generations. Monitoring prescribing trends in our hospital as a means of identifying targets for improving prescribing is inevitable.

Objective: The aim of the study was to evaluate antibiotic prescribing practices for hospitalised children with suspected bacterial infections in a Paediatric hospital in Nigeria.

Methods: A retrospective survey was carried out using case notes of previously hospitalised patients admitted between January and June 2016. Data from 150 case notes of patients admitted for suspected bacterial infections were collected using a predesigned data collection form. Patients' demographics, infection type, details of prescribed antibiotics, length of hospital stay and microbiological assessments were noted. Data were analysed using statistical package for social sciences (SPSS) version 22. Frequencies and percentages were calculated for categorical variables. Means and standard deviations were calculated for continuous (numerical) variables. Correlation was also employed in the analysis.

Results: Of the 150 patients, $53.3 \%$ were males and $86 \%$ were children under 5 years of age. The mean duration of hospital stay was 7.59 ( \pm 5.4 ) days. The most common infections were respiratory tract infection (32\%) and sepsis $(31.3 \%)$. The most common empirically prescribed antibiotics at the onset of admission were Gentamicin and a fixed dose combination of Ampicillin/Cloxacillin which were prescribed for $64.7 \%$ and $52.7 \%$ of the patients respectively. Cultures were ordered for only 7 (4.7\%) of patients at the onset of hospitalisation. All antibiotics administered on admission were parenteral formulations and only $4 \%$ of the patients had their antibiotic switched to oral route on or before the third day of patients' admission. Another 71.3\% were converted to oral formulations on the day of discharge from the hospital. A total of $87.3 \%$ were discharged on antibiotics and the most commonly prescribed antibiotic at discharge was Cefixime (37.2\% of antibiotics prescribed as take home medication). Conclusions: Antibiotics were started empirically in all cases and cultures were ordered for few patients at the start of antibiotic therapy. Cultures should be more frequently ordered in the hospital to guide antibiotic prescribing for patients admitted for suspected bacterial infections. In addition, timely intravenous (IV) to oral (PO) antibiotic switch should be practised whenever appropriate. Educating physicians on the benefits of early switch from IV to PO formulations when appropriate is also recommended. Initiatives such as the "Antibiotic Time out" or Start Smart-then Focus approach will be appropriate in the hospital. Introduction of an empiric antibiotic policy in the hospital is highly recommended.
\end{abstract}

Key Words: Antibiotic prescribing, Antibiotic resistance, Hospitalised children

*Correspondence: Nneka Egbuchulam; Email: negbuchulam@gmail.com; Address: Department of Clinical Pharmacy and Biopharmacy, Faculty of Pharmacy, University of Lagos, Nigeria. 


\section{INTRODUCTION}

Antibiotics are among the most commonly prescribed drugs in paediatrics and they form a critically important part of paediatric medical care in low and middle income countries (LMICs) where infectious diseases are the leading cause of child mortality. ${ }^{[1]}$ Undoubtedly, many children especially those living in developing countries such as Nigeria have benefited from the use of antibiotics against infectious diseases. Sadly, antibiotic resistance (ABR) is now an issue of worldwide concern to all stakeholders including policy makers.

Infants and children are among the most vulnerable groups in the contraction of illness, routine use of antibiotics and subsequent ABR. ${ }^{[2]}$ According to ReAct (Action on ABR), neonates and children are a major group affected by $A B R .^{[3]}$ In addition, recent findings from a United States study show that antibiotic resistant infections are on the increase even among children. ${ }^{[4]}$ The problem of ABR is particularly prevalent in resource limited countries and is fuelled by irrational antibiotic prescribing. ${ }^{[5]}$ The consequences not only affect patients' lives but also go beyond the individual patient, affecting healthcare systems and societies around the world. ${ }^{[3]}$

Antibiotics are frequently prescribed irrationally in terms of type, dose, duration and indication. ${ }^{[6]}$ Optimal prescribing of antibiotics is the first step in reducing ABR, which implies that antibiotics must be appropriately used and regulated in all hospital settings. ${ }^{[7]}$ To achieve this, hospitals need to ensure that the most appropriate antibiotics are selected in correct doses for appropriate duration of time and in a manner that does not encourage the development of resistant strains.

It is important to note that robust data on the use of antibiotics are lacking for hospitalised children. ${ }^{[8]}$ According to Irwin and Sharland, ${ }^{[9]}$ little is known about the exposure to antibiotics in hospitalised children, particularly in resource poor countries where the burden of infectious disease is highest. It is therefore important to generate such data through studies of antibiotic use in children. In addition, it is a pivotal step for improving the use of antibiotics and ultimately patient outcome in this vulnerable population.

Broad spectrum antibiotics are often the empiric drugs of choice in the management of infections especially for hospitalised patients. ${ }^{[10]}$ These may be appropriate as the causative agents may not be known. However prescribers should be more aware that a more broad spectrum empirical therapy does not result in a more effective treatment, but does increase the possibility of ABR. ${ }^{[11]}$ Modifications of empirical antibiotic therapy when warranted by culture results or clinical signs are recommended to control antimicrobial overuse

Published by Sciedu Press and resistance. ${ }^{[12]}$ One of the 12 step strategies for reducing antimicrobial resistance in hospitalised children includes the need to target empiric antimicrobial therapy to likely pathogens and also to target definitive antimicrobial therapy to known pathogens through de-escalation or escalation. ${ }^{[13]}$ Culture and sensitivity testing should help physicians modify antibiotic therapy prescribed for patients at the onset of hospitalisation. Discontinuation of ceftriaxone in $30 \%$ of cases after negative blood culture was identified as an indication to rational antibiotic use in patients admitted in a Pakistan hospital. ${ }^{[14]}$

Parenteral antibiotic use is seemingly common among hospitalised paediatric patients especially at the onset of admission. The extent to which early switch from parenteral to oral antibiotic formulations is carried out should be verified. Considering the paucity of data on antibiotic prescribing for hospitalised patients in Nigeria, this study sought to assess antibiotic prescribing specifically for inpatients admitted with a suspected bacterial infection diagnosis in a paediatric hospital in Nigeria.

\section{Methods}

A retrospective study was carried out at a free standing specialist hospital for children located in a densely populated part of Lagos Island in Lagos State, Nigeria. The hospital offers a wide range of services for neonates and paediatric patients and also has a functioning intensive care unit for neonates. Permission for this study was obtained from the Health Service Commission of the Lagos State Ministry of Health, Lagos Nigeria.

The survey was carried out using case notes of previously hospitalised patients admitted between January and June 2016. The case notes were retrieved from the health records department of the hospital. One of the investigators (a postgraduate pharmacist) studied these case notes and identified patients based on specified inclusion criteria (patients admitted for a suspected infection and commenced antibiotic on the day of admission). Patients admitted for other reasons other than for an infection or those with a length of stay less than three days were excluded from the survey. A predesigned data collection form was used to collect the following details from the case notes: patients' demographics, duration of hospital stay, diagnosis, diagnostic information such as fever, white blood cell (WBC) count and whether patient was concurrently treated for malaria. Information on prescribed antibiotic(s) on admission and practice of intravenous (IV) to oral (PO) switch on or before third day of hospital admission was noted. Results of microbiological evaluation and subsequent adjustment to antibiotic therapy following positive cultures (if any) was also documented. Antibiotics given as 
take home medication were also recorded.

Data were analysed using SPSS version 22. Frequencies and percentages were calculated for categorical variables. Means and standard deviations were calculated for continuous (numerical) variables. Correlation was also employed in the analysis.

\section{RESUlts}

Table 1 reflects the demographic and clinical characteristics of patients. Of the 150 patients, the proportion of males to females was $53.3 \%: 46.7 \%$. The patients were predominantly paediatric patients $83.3 \%$ (age $>28$ days), while $16.7 \%$ were neonates ( $\leq 28$ days). Overall $86 \%$ were children under 5 years of age. Findings also show that $51.3 \%$ of patients had fever on admission (defined as temperature over $38^{\circ} \mathrm{C}$ ). The first white blood cell count value at the onset of hospitalisation was considered to be normal in $40.7 \%$ of patients and abnormal in $59.3 \%$ (Normal WBC count defined as more than 4,000 or less than 12,000 cells per $\mu \mathrm{l})$. Slightly less than half of the patients $(47.3 \%)$ had positive malaria parasite blood test and were subsequently treated for malaria. The mean duration of hospital stay was 7.59 ( \pm 5.4$)$ days. Of the 150 admitted patients, 46 (30.7\%) were referred from other hospitals. Previous antibiotic use either from referred hospital or through self-medication was documented in case notes of $38 \%$ of all the hospitalised patients.

Table 1. Socio-demographic and clinical data on admission

\begin{tabular}{lll}
\hline Characteristics & Sub-group & Number (\%) \\
\hline \multirow{2}{*}{ Sex } & Male & $80(53.3)$ \\
& Female & $70(46.7)$ \\
& $\leq 28$ days & $25(16.7)$ \\
Age of child & 29 days - 12 months & $58(38.7)$ \\
& $>1$ year-5 years & $46(30.7)$ \\
& $>5$ years-12 years & $19(12.7)$ \\
& $>12$ years & $2(1.3)$ \\
Length of stay in & $>3-7$ & $101(67.3)$ \\
days & $8-14$ & $38(25.3)$ \\
& $15-21$ & $7(4.7)$ \\
Fever & $>21$ & $4(2.7)$ \\
White blood cell & Fever & $77(51.3)$ \\
Infections with & No Fever & $73(48.7)$ \\
comorbid malaria & Ybnormal & $61(40.7)$ \\
& No & $89(59.3)$ \\
& Respiratory Tract Infections & $71(47.3)$ \\
Specific infections & Central Nervous System & $19(26.8)$ \\
with malaria & Sepsis & $18(25.3)$ \\
& Gastrointestinal & $17(23.9)$ \\
& Others & $6(115.5)$ \\
\hline
\end{tabular}

Details of the most common suspected infections were in- fections of the respiratory tract (32\%), sepsis (31.3\%) and central nervous system $(17.3 \%)$ respectively as shown in Figure 1. For respiratory tract infections, pneumonia was the most occurring, comprising $81.3 \%$ of all respiratory tract infections. The most commonly prescribed antibiotic for the treatment of respiratory infections were a combination of cefuroxime and gentamicin, which were prescribed for $31.3 \%$ of patients. A combination of ampicillin/cloxacillin and gentamicin were commonly prescribed for $51.1 \%$ of patients with sepsis while ceftriaxone was the most commonly prescribed antibiotic (42.3\% of patients) for infections of the central nervous system as shown in Table 2.

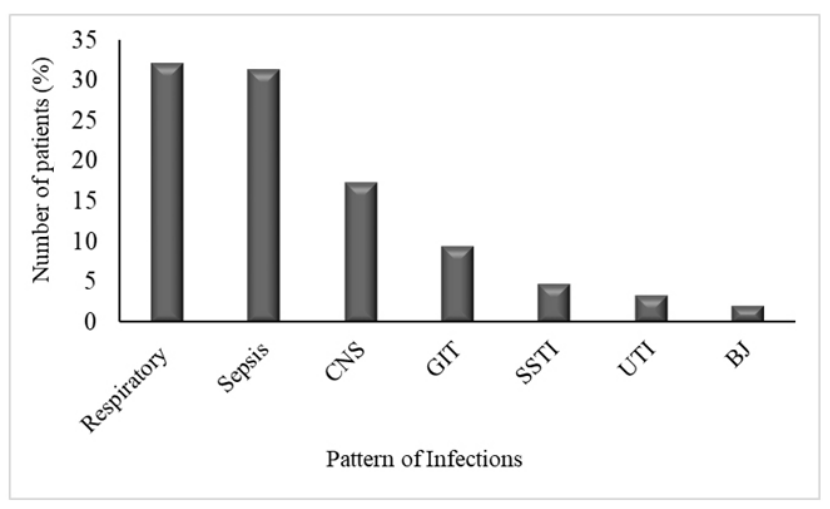

Figure 1. Distribution of types of infections among hospitalised children

$C N S=$ Central nervous system, UTI = Urinary tract infection, GIT $=$ Gastrointestinal tract, $S S T I=$ Skin and soft tissue infection, BJ = Bone and Joint infections

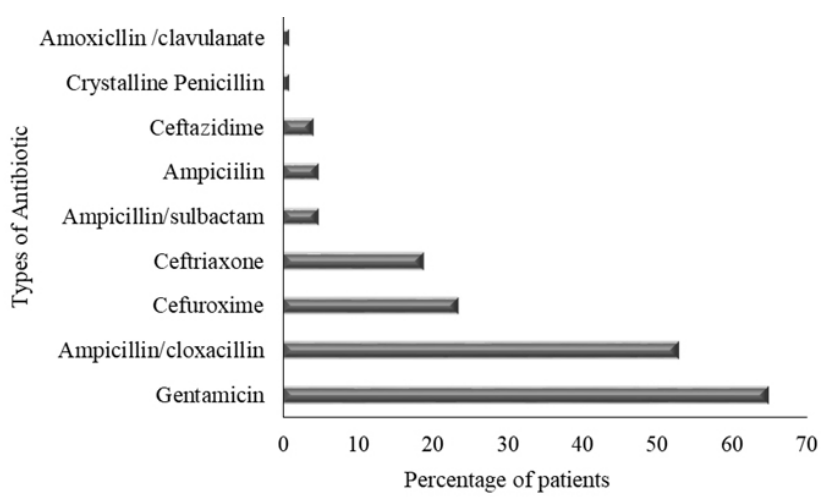

Figure 2. Percentage of patients on different Antibiotics empirically prescribed at the onset of hospitalisation

At the onset of admission, 251 antibiotic courses were prescribed for 150 patients. Overall, a total of 9 different antibiotic types were prescribed alone or in combination for suspected infections, with gentamicin and ampicillin/cloxacillin respectively being prescribed for $64.7 \%$ and $52.7 \%$ of patients at the onset of hospitalisation (see Figure 2). 
Table 2. Type and number of antibiotics prescribed for different infections

\begin{tabular}{|c|c|c|}
\hline Diagnosis/number of patients with condition & Antibiotic treatment given & Number of patients (\%) \\
\hline \multirow{5}{*}{ Respiratory Tract Infections (48) } & Cefuroxime + gentamicin & $15(31.3)$ \\
\hline & Ampicillin/cloxacillin + gentamicin & $18(37.5)$ \\
\hline & Cefuroxime & $9(18.8)$ \\
\hline & Ampicillin/cloxacillin & $4(8.3)$ \\
\hline & Ceftriaxone & $2(4.2)$ \\
\hline \multirow{7}{*}{ Sepsis (47) } & Ampicillin/cloxacillin + gentamicin & $24(51.1)$ \\
\hline & Ampicillin/cloxacillin & 7 (14.9) \\
\hline & Ampicillin/sulbactam + gentamicin & 7 (14.9) \\
\hline & Ceftazidime + gentamicin & $6(12.8)$ \\
\hline & Ceftriaxone & $1(2.1)$ \\
\hline & Ceftriaxone + gentamicin & $1(2.1)$ \\
\hline & Cefuroxime + gentamicin & $1(2.1)$ \\
\hline \multirow{6}{*}{ Central Nervous System (26) } & Ceftriaxone & $11(42.3)$ \\
\hline & Ceftriaxone + gentamicin & $6(23.1)$ \\
\hline & Ampicillin/cloxacillin + gentamicin & $4(15.4)$ \\
\hline & Ceftriaxone + ampicillin & $3(11.5)$ \\
\hline & Ampicillin cloxacillin & $1(3.8)$ \\
\hline & Ceftriaxone + crystalline penicillin & $1(3.8)$ \\
\hline \multirow{4}{*}{ Gastrointestinal (14) } & Ampicillin/cloxacillin + gentamicin & $6(42.9)$ \\
\hline & Ampicillin/cloxacillin & $4(28.6)$ \\
\hline & Cefuroxime & $3(21.4)$ \\
\hline & Amoxicillin/ clavulanate & $1(7.1)$ \\
\hline \multirow{3}{*}{ Urinary tract (5) } & Cefuroxime + gentamicin & $2(40)$ \\
\hline & Ampicillin/cloxacillin + gentamicin & $2(40)$ \\
\hline & Ceftriaxone & $1(20)$ \\
\hline \multirow{4}{*}{ Skin and soft tissue (7) } & Cefuroxime & $4(57.1)$ \\
\hline & Ceftriaxone & $1(14.3)$ \\
\hline & Cefuroxime + gentamicin & $1(14.3)$ \\
\hline & Ampicillin/cloxacillin + gentamicin & $1(14.3)$ \\
\hline \multirow{2}{*}{ Bone and Joint (3) } & Ampicillin/cloxacillin + gentamicin & $2(66.7)$ \\
\hline & Ceftriaxone + gentamicin & $1(33.3)$ \\
\hline
\end{tabular}

Antibiotic prescription characteristics at the onset of admission and during the entire period of hospital stay is shown in Table 3. A total of 349 antibiotic courses were prescribed for the entire duration of hospital stay. Individual patients received between 1 and 6 antibiotics during their hospital stay and mean number of antibiotic per patient was 2.33 .

Of the 150 patients admitted on the basis of a possible infection, cultures were ordered for $7(4.7 \%)$ patients within the first day of admission and none of the cultures was subsequently reported positive. However, for the entire duration of hospital stay, cultures were ordered for 19 (12.7\%) of the patients, with cerebrospinal fluid and blood cultures accounting for $52.6 \%$ and $31.6 \%$ of all cultures respectively. Out of all

ordered cultures done during the entire hospital stay, only 2 $(10.5 \%)$ yielded positive growth. One blood culture yielded Staphylococcus spp. and the second yielded a mixed growth of Klebsiella spp. and Pseudomonas aeruginosa. Positive culture results did not lead to any change in the antibiotic course administered at the time culture results were available. In one of the patients however, the sensitivity profile of the isolated organism corresponded to the choice of the already prescribed antibiotic for the patient.

All patients (100\%) were administered IV antibiotics on admission and only $4 \%$ of patients had their prescription switched to the oral route by the third day of therapy, while another $71.3 \%$ had their antibiotic converted to the oral route 
on the day of discharge as part of the patients discharge plan from hospital. There was a very high positive significant correlation $(r=0.951, p<.001)$ between length of IV antibiotics therapy and duration of hospital stay, as it was observed that the duration of IV therapy increased with duration of hospital stay and vice versa. Also observed was that 62 (41.3\%) patients had missed at least one dose of prescribed antibiotic during their hospital stay. Although ceftriaxone was the fourth most commonly prescribed antibiotic (see Figure 2), it was the most commonly missed medication, accounting for $37.9 \%$ of missed doses. Overall, $87.3 \%$ of the patients were discharged on antibiotics and the three most commonly prescribed take home antibiotic medications were cefixime (37.2\%), cefuroxime (27\%) and ampicillin/cloxacillin combination $(18.2 \%)$.

Table 3. Antibiotic prescription characteristics

\begin{tabular}{lll}
\hline Prescription characteristics & Sub-group & Number (\%) \\
\hline Number of initial antibiotics prescribed for patients at onset of & 1 & $49(32.7)$ \\
hospitalisation & 2 & $101(67.3)$ \\
& 1 & $36(24)$ \\
& $28(38.7)$ \\
Total number of antibiotics prescribed during the entire hospital stay & 3 & $38(25.3)$ \\
& 4 & $10(6.7)$ \\
& 5 & $7(4.7)$ \\
IV to PO conversion within first three days of hospitalisation & 6 & $1(0.7)$ \\
IV to PO conversion on day of discharge & Yes (4) \\
Discharged on antibiotic & No & $144(96)$ \\
& Yes & $107(71.3)$ \\
Missed antibiotic doses during hospitalisation & No & $32(21.3)$ \\
\hline
\end{tabular}

\section{Discussion}

From the study, respiratory tract infection and sepsis showed the highest frequencies of occurrence. This is similar to a study carried out in Tanzania ${ }^{[15]}$ where both conditions were also identified as the most common reasons for hospitalisation. Amongst respiratory infections, pneumonia was observed to be the most frequent infection for which antibiotics were prescribed. Similar studies in Ethiopia and Tanzania showed that pneumonia was implicated as the most common reason for hospitalisation. ${ }^{[16-19]}$ This implies that pneumonia is a major cause of morbidity and hospitalisation among children in these African countries.

The most commonly prescribed antibiotics in this study were gentamicin and a fixed dose combination (FDC) of ampicillin/cloxacillin which were prescribed for $64.7 \%$ and $52.7 \%$ of patients respectively. Antibiotic selection in this hospital is possibly influenced by the clinical guidelines of the hospital which advocates the use of ampicillin/cloxacillin in combination with an aminoglycoside (gentamicin) for infections such as pneumonia and sepsis.
Findings from other studies in similar patient populations reported ampicillin and gentamicin as the most commonly prescribed antibiotics. ${ }^{[15,18]}$ while ceftriaxone and gentamicin were the most common in two Ethiopian hospitals. ${ }^{[16,19]}$ In the hospital investigated, we observed that ceftriaxone was the fourth commonly prescribed antibiotic and the most often prescribed for patients with meningitis. It appears that the use of ceftriaxone in this hospital is selective as findings from other studies identified ceftriaxone as the most common antibiotic prescribed in their hospitals. ${ }^{[14,17,19,20]}$

Ampicillin/cloxacillin combination was also observed to be the second most commonly prescribed antibiotic in the hospital. This fixed dose combination (FDC) was also reportedly used in Nepal ${ }^{[21]}$ and Indian ${ }^{[22]}$ hospitals. However, Poudel et al. ${ }^{[23]}$ opined that contrary to its wide spread use, data regarding the justification for the use of this combination were lacking. Although, the British National Formulary (BNF) is widely used in Nigeria, this FDC ampicillin and cloxacillin is not mentioned or referenced in the BNF for children (BNFC). ${ }^{[24]}$ This is in line with Poudel's statement 
that worldwide accepted drug information sources like the BNF and the United States Pharmacopoeia Drug Information (USPDI) did not approve this FDC. ${ }^{[23]}$

Of the 150 patients treated for a suspected infection, $71(47.3 \%)$ of these patients were treated with an antimalarial during their hospital stay. Malaria was described as a driver of antibiotic use in Zambia and Uganda study. ${ }^{[25]}$ However in this hospital appropriate test to support the administration of an antimalarial during their hospital stay was carried out. This was informed by the fact that Nigeria is a malaria endemic country, it is therefore likely that infections and malaria could co-exist during the period of hospitalisation especially in children. A total of $59.3 \%$ of patients had abnormal WBCs, suggestive of the presence of an infection in patients who had a minimum length of stay of 3 days. However, the percentage of cultures ordered at the beginning of therapy appears low, though sepsis (either in neonatal or paediatric patients) accounted for $31.3 \%$ of hospitalised patients in this hospital. It is important to note that the Surviving Sepsis Campaign Guideline, 2012 recommends obtaining appropriate cultures before antimicrobial therapy is initiated if such cultures do not cause significant delay in the start of antimicrobials. The rationale is that this practice is essential to confirm infection as well as the responsible pathogen, and to allow for de-escalation of antimicrobial therapy after receipt of susceptibility profile. ${ }^{[26]}$

In Pakistan, findings from a study of 131 hospitalised children reported that blood cultures were done for 114 (87\%) although only 10 cases $(8.8 \%)$ were found to be culture positive. ${ }^{[14]}$ Palikhe ${ }^{[21]}$ reported that among 121 patients clinically diagnosed with infectious diseases, specimens were taken for cultures in only 24 cases (19.8\%), out of which 13 specimens (54.2\%) showed positive culture results. Another study reported that doctors in two healthcare facilities did not routinely send samples for microbiological testing, implying that there was no laboratory confirmation performed to adjust the initial empiric antibiotic therapy. ${ }^{[22]}$ In another study, antimicrobial treatment was guided by the results of laboratory tests in just $14.3 \%$ of cases, which the authors described as far from satisfactory. ${ }^{[27]}$

In the current study, only 7 (4.7\%) and 19 (8.67\%) patients had cultures at the beginning and during the whole duration of hospitalisation respectively. None of the cultures done at the onset of hospitalisation yielded any growth while only 2 of the 19 cultures done during the entire period of stay yielded positive growth. Badiya et al. ${ }^{[27]}$ also reported the low yield of culture tests and stated that this scenario often frustrates clinicians. In our study, $38 \%$ of patients already had prior antibiotic use as stated in patients' case notes.
Exposure to antibiotics prior to hospitalisation could compromise culture results. Palikhe ${ }^{[21]}$ however emphasised the importance of ensuring that appropriate cultures are ordered before initiation of antibiotics. Use of cultures as a means of ensuring a more definitive approach to antibiotics prescribing should be encouraged possibly through the development and implementation of an Empiric Antibiotic Policy in the hospital.

Another finding in this study was that IV to PO conversion occurred in only $4 \%$ of patients by the third day of therapy. It appeared that the practice of conversion was more often implemented on the day of discharge as part of patient's discharge plan. In another study, Feleke et al. ${ }^{[16]}$ reported that parenteral to oral shift was not practised at all for hospitalised paediatric patients and suggested that the issue needed urgent solution. This observation is similar to the findings from another study in paediatric patients where the authors called for increasing professionals' awareness about antibiotics particularly the benefit of parenteral to oral conversion. ${ }^{[19]}$ The practice of delaying IV to PO conversion till the day of discharge is very likely to contribute to an extension of antibiotic duration, increased cost of medications, more chances of experiencing adverse reactions or even infections associated with the use of IV lines.

The practice of IV to PO switch should be implemented, keeping in view the patients' clinical status including the ability to tolerate oral medications, which could be a challenge particularly for neonates. However opportunities for switching when appropriate and not necessarily on the day of patients discharge as (observed in this study) should be considered. Specifically, stepping down to the appropriate oral antibiotic when improvement is sustained is recommended in the guidelines for management of Community Acquired Pneumoniae (CAP) in Nigeria. ${ }^{[28]}$ In addition, the review of choice, dose and route of administration of prescribed antibiotic at certain time points (48-72 hours) as recommended by the Centers for Disease Control and Prevention (CDC) and other reputable global authorities should be encouraged. ${ }^{[29,30]}$ Antibiotic Time out ${ }^{[29]}$ and Start SmartThen Focus ${ }^{[30]}$ approach are examples of such initiatives prompting physicians to perform reviews with the aim of taking certain decisions including the possibility of adjusting the route of administration from IV to PO route. The Start Smart-then focus approach also emphasises the importance of ordering cultures at the start of empirical antibiotic prescribing.

Missed doses to prescribed antibiotics was observed as a challenge to rational antibiotic use in the hospital. A total of $41.3 \%$ of the patients had at least one incident of missed 
dose recorded in their drug chart for the entire duration for which antibiotics were prescribed during their hospital stay. Another study in the United Kingdom reported that $13.2 \%$ of patients missed one or more prescribed antimicrobial doses over a 24-hour period while on hospital admission. ${ }^{[31]}$ Findings from Uganda also reported frequent missed doses of antibiotics among hospitalised patients of which ceftriaxone was among the most frequently missed antimicrobial. ${ }^{[32]}$

In the current study, it was observed that some of the reasons for missed doses in patients admitted with a length of stay of over three days were unavailability and unaffordability of drugs. These are in line with the report of Mezgebe et al. ${ }^{[17]}$ that paediatricians and other medical personnel who provide healthcare for infants and children in developing countries are faced with numerous challenges due to the shortage of appropriate drugs, costs and lack of infrastructure. These give cause for concern as the aim of antibiotic stewardship is to ensure that the right patient is given the right antibiotic at the right time for the right duration. In spite of these obvious challenges, measures that will encourage the appropriate selection and use of antibiotics in our hospitals should be considered and implemented.

\section{Conclusions}

Empiric antibiotic prescribing for patients admitted in the hospital was mainly gentamicin and a fixed dose combination of ampicillin/cloxacillin, while the use of ceftriaxone appears to be selective. Ordering of samples for microbiological evaluation at the onset of hospitalisation was observed to be low. Efforts targeted at timely ordering of cultures among physicians should be encouraged as a means of achieving definitive antibiotic prescribing.
IV to PO conversion occurred in only $4 \%$ of patient by the third day of therapy. Most patients were switched to oral therapy on the day of discharge, a trend which is likely to increase length of antibiotic use and hospital stay. Encouraging timely parenteral to oral conversion of antibiotics when appropriate and not necessarily on the day of patient's discharge is also recommended. Controlling the use of parenteral antibiotics will be a major step to controlling medication cost in a resource limited country like Nigeria. Educating physicians on the benefits of early switch from IV to PO formulations when appropriate is also recommended. Initiatives such as the "Antibiotic Time out" or Start Smart-Then Focus approach will be very appropriate in the hospital. Introduction of an empiric antibiotic policy in the hospital is highly recommended.

\subsection{Strengths}

The study provides insight into prescribing practices for children and identifies possible areas for improving prescribing in a free standing children hospital in Lagos, Nigeria. It was helpful in providing a realistic assessment of antibiotic prescribing which might have been possibly influenced if the survey had been conducted prospectively in the wards.

\subsection{Limitations}

The use of only one hospital in Nigeria limits the robustness of the result, which can therefore not be generalised.

\section{ACKnOWledgements}

The authors acknowledge the hospital management for their support during the study period.

\section{Conflicts of InTEREST Disclosure}

The authors declare they have no conflicts of interest.

\section{REFERENCES}

[1] Le Doare K, Barker CI, Irwin A, et al. Improving antibiotic prescribing for children in the resource-poor setting. British Journal of Clinical Pharmacology. 2014; 79(3): 446-455. PMid: 24433393. https://doi.org/10.1111/bcp. 12320

[2] Medscape. Antibiotic Resistance Continues to be a Problem in Children. March 23, 2016 [18 March 2017, date last accessed]. Available from: http://www.medscape.com/viewarticle/860801

[3] ReAct-Action on Antibiotic Resistance. Burden of Antibiotic Resistance. 2012 [7 June 2016, date last accessed]. Available from: https://www.reactgroup.org/uploads/publications/re act-publications/ReAct-facts-burden-of-antibioti c-resistance-May-2012.pdf

[4] Centre for Diseases Dynamics, Economics and Policy (CDDEP). Antibiotic resistant infections on the rise among children in the US, study finds. March 20, 2014 [18 March 2017, date last accessed]. Available from: https://cddep.org/blog/posts/antibiotic_resist ant_infections_rise_among_children_us_study_finds/

[5] Thriemer K, Katuala Y, Batoko B, et al. Antibiotic prescribing in DR Congo: a knowledge, attitude and practice survey among medical doctors and students. PLoS ONE. 2013; 8(2): e55495. PMid: 23441152. https ://doi.org/10.1371/journal.pone.0055495

[6] Sutradhar KB, Saha A, Huda NH, et al. Irrational Use of Antibiotics and Antibiotic Resistance in Southern Rural Bangladesh; Perspectives from both the physicians and patients. Annual Research \& Review in Biology. 2014; 4(9): 1421-1430. https://doi .org/10 $.9734 / \mathrm{ARRB} / 2014 / 8184$

[7] Alahmadi Y, Aldeyab M, Scott M, et al. Current patterns of antibiotic use and the prevalence of hospital-acquired infection (HAI) in hospitalized patients within hospital setting in Northern Ireland. Hospital Pharmacy. 2016 [10 November 2017, date last accessed]. Available from: http: //www.hospitalpharmacyeurope.com/featured-articles/ point-prevalence-surveys-antibiotic-use-and-hais 
[8] Gharbi M, Doerholt K, Vergnano S, et al. Using a simple point prevalence survey to define appropriate antibiotic prescribing in hospitalised children across the UK. BMJ Open. 2016; 6: e012675. PMid: 27810974. https://doi.org/10.1136/bmjopen-2016-01267 5

[9] Irwin A, Sharland M. Measuring antibiotic prescribing in hospitalised children in resource-poor countries. A systematic review. Journal of Paediatric and Child Health. 2013; 49: 185-192. PMid: 21679337. https://doi.org/10.1111/j.1440-1754.2011.02126.x

[10] Leeka S, Terrell CL, Edson R. General principles of Antimicrobial therapy. Mayo Clinic Proceedings. 2011; 86(2): 156-167. PMid: 21282489. https://doi.org/10.4065/mcp. 2010.0639

[11] Van Spreuwel PCJM, Blok H, Langelaar MFM, et al. Identifying targets for quality improvement in hospital antibiotic prescribing. The Netherlands Journal of Medicine. 2015; 73(4): 161-168. PMid: 25968287.

[12] Braykov NP, Morgan DJ, Schweizer ML, et al. Assessment of empirical antibiotic therapy optimization in six hospitals: an observational cohort study. Lancet Infect Disease. 2014; 14: 1220-1227. https://doi .org/10.1016/S1473-3099(14)70952-1

[13] Centre for Disease Control and Prevention (CDC) Campaign to prevent antimicrobial resistance in healthcare settings: 12 steps strategy to prevent antimicrobial resistance among hospitalized children. 2013 [15 July 2016, date last accessed]. Available from: http://www.kliinikum.ee/infektsioonikontrolli teenistus/doc/oppematerjalid/children.pdf

[14] Ali SR, Ahmed S, Lohana H. Trends of empiric antibiotic usage in a secondary care hospital, Karachi, Pakistan. International Journal of Paediatrics. 2013; 2013: 832857. https://doi.org/10.1155/20 $13 / 832857$

[15] Wojt S. The use of antibiotics at two paediatric wards at Kilimanjaro Christian Medical Centre (KCMC) in Moshi, Tanzania. Masters thesis. 2014. [10 December 2016, date last accessed]. Available from: https://gupea.ub.gu.se/handle/2077/37165https: //gupea.ub.gu.se/bitstream/2077/37165/1/gupea_2077 _37165_1.pdf

[16] Feleke M, Yenet W, Lenjisa JK. Prescribing pattern of antibiotics in peadiatric wards of Bishoftu Hospital, East Ethiopia. International Journal of Basic \& Clinical Pharmacology. 2013; 2(6): 718-722. https://doi.org/10.5455/2319-2003.ijbcp20131209

[17] Mezgebe HB, Tadesse B, Legesse B. Antibiotics prescribing pattern in pediatric unit of Ayder Referral Hospital, Tigray Region, Northern Ethiopia. Journal of Scientific and Innovative Research. 2015; 4(2): 57-60.

[18] Alemnew G, Atnafie SA. Assessment of the pattern of antibiotics use in pediatrics ward of Dessie Referral Hospital, North East Ethiopia. International Journal of Medicine and Medical Sciences. 2015; 7(1): 1-7. https://doi.org/10.5897/IJMMS2014.1101

[19] Achalu T, Mensa M. Retrospective drug use pattern of antibiotics in pediatric ward of Shenan Gibe Hospital, Oromia Region, Ethopia. Journal of Antibiotic Research. 2017; 1(1): 106.

[20] Gopal MB, Thiyagarajan P, Venugopal V, et al. A study on antibiotic prescription among the hospitalized pediatric patients at a referral center in Puducherry, India. International Journal of Contemporary Pediatrics. 2017; 4(3): 700-705. https : //doi.org/10.18203/2 349-3291 . i jcp20170922

[21] Palikhe N. Prescribing pattern of antibiotics in Pediatric Hospital of Kathmandu Valley. Journal of Nepal Health Research Council. 2004; 2(2): 31-36.

[22] Sharma M, Damlin A, Pathak A, et al. Antibiotic prescribing among pediatric inpatients with potential infections in two private sector hospitals in Central India. PLoS ONE. 2015; 10(11): e0142317. PMid: 26540104. https://doi.org/10.1371/journal.pone .0142317

[23] Poudel A, Subish P, Mohammed I. Use of ampicillin/cloxacillin combination in Nepal: need for intervention. Journal of Gandaki Medical College Nepal. 2008; 1: 61-63.

[24] British National Formulary for Children. London: British Medical Journal Group, Royal Pharmaceutical Society, Royal College of Paediatrics and Child Health, and Neonatal and Paediatric Pharmacists Group. 2014

[25] Foster SD, Sosa A, Najjuka CF, et al. Drivers of antibiotic resistance in Uganda and Zambia. Presentation to the Global Health Council. Washington, DC. 14 June 2011 [15 August, 2015, date last accessed]. Available from: http://emerald.tufts.edu/med/apua/resea rch/gates_19_970229113.pdf

[26] Dellinger RP, Levy MM, Rhodes A, et al. Surviving Sepsis Campaign: International Guidelines for Management of Sepsis and Septic Shock: 2012. Critical Care Medicine. 2013; 41(2): 580-637. PMid: 23353941. https://doi.org/10.1097/CCM.0b013e31827e83 af

[27] Baidya S, Hazra A, Datta S, et al. A study of antimicrobial use in children admitted to pediatric medicine ward of a tertiary care hospital. Indian Journal of Pharmacology. 2017; 49: 10-5. PMid: 28458416.

[28] Olowu A, Elusiyan JBE, Esangbedo D et al. Management of community acquired pneumonia (CAP) in children: clinical practice guidelines by the Paediatrics Association of Nigeria. Niger J Paed. 2015; 42(4): 283-292. https://doi.org/10.4314/njp.v42i4.1

[29] CDC Core Elements of Hospital Antibiotic Stewardship Programs. 2012 [10 September 2015, date last accessed]. Available from: https://www.cdc.gov/antibiotic-use/healthcare/ implemnetation/core-elements.html

[30] Ashiru-Oredope D, Sharland M, Charani E, et al. Improving the quality of antibiotic prescribing in the NHS by developing a new Antimicrobial Program: Start Smart-Then Focus. Journal of Antimicrobial Chemotherapy. 2012; 67: i53-i63. PMid: 22855879. https://doi.org/10.1093/jac/dks202

[31] Wright J. Audit of missed or delayed antimicrobial drugs. Nursing Times. 2013; 109(42): 11-14. PMid: 24288859.

[32] Kiguba R, Karamagi C, Bird SM. Extensive antibiotic prescription rate among hospitalized patients in Uganda: but with frequent misseddose days. Journal of Antimicrobial Chemotherapy. 2016; 71(6): 1697-706. PMid: 26945712. https://doi.org/10.1093/jac/ dkw025 